\section{Dialyse erhöht Risiko für Nieren- und Schilddrüsenkarzinom}

\begin{abstract}
Bei Patienten mit einem dialysepflichtigen Nierenversagen ist die Wahrscheinlichkeit für eine Krebserkrankung erhöht, wie eine US-Studie belegt. Dabei gibt es bei den Krebsarten offenbar Unterschiede in der Inzidenz.
\end{abstract}

U $\mathrm{m}$ die Zusammenhänge zwischen dialysepflichtigem Nierenversagen und verschiedenen Tumorarten zu klären, führten Elizabeth L. Yanik et al. vom National Cancer Institute, Bethesda, USA, eine große Registerstudie durch. Dafür werteten sie Befunde von mehr als 202.000 Patienten mit Nierenversagen, die für eine Transplantation vorgesehen waren, sowie von Transplantatempfängern aus.

Yanik et al. betrachteten die Phase, in der die Patienten auf der Warteliste standen und dialysiert wurden, die Zeit nach der ersten Transplantation, wenn immunsupprimiert wurde, die Phase der Transplantatabstoßung sowie die entsprechenden Phasen nach weiteren Transplantationen und möglichem Ver- sagen der Spenderorgane. Dadurch konnten die Ärzte Krebsinzidenzen den Therapiemaßnahmen wie Dialyse und Immunsuppression gezielt zuordnen.

Es stellte sich heraus, dass Patienten, die dialysiert werden müssen, offenbar ein erhöhtes Risiko haben, an einem Nierenzell- oder Schilddrüsenkarzinom zu erkranken. Die Wahrscheinlichkeit für diese Tumoren ist in der Gruppe der Patienten mit intaktem Transplantat im Vergleich zu Patienten auf der Warteliste (mit Dialyse) und denjenigen mit einem Transplantatversagen signifikant verringert (Hazard Ratio [HR] für Nierenkrebs: 0,77, $95 \%$-Konfidenzintervall [95\%-KI] zwischen 0,7 und 0,84; $<<0,001$; HR für Schilddrüsenkrebs: 0,67; 95\%-KI zwischen 0,56 und 0,$81 ; \mathrm{p}<0,001)$.
Unter Immunsuppression war unter anderem das Risiko für Kaposi-Sarkome, Non-Hodgkin- und Hodgkin-Lymphome signifikant höher als etwa bei Patienten auf der Warteliste für die Nierentransplantation.

Fazit: Dass Patienten mit Hämodialyse ein erhöhtes Krebsrisiko haben, bestätigte auch eine aktuelle US-amerikanische Studie [Am J Kidney. 2015;65:76372]. Von mehr als 482.000 Dialysepatienten erkrankten innerhalb von fünf Jahren nach Beginn der Therapie mehr als 37.000 an Krebs. Die kumulative Fünf-Jahres-Inzidenz für jegliche Tumorart betrug 9,48\% (95\%-KI zwischen 9,39\% und 9,57\%). Vor allem für die Entstehung eines Nieren- und Nierenbeckenkarzinoms sowie Harnblasenkarzinoms war das Risiko erhöht.

Peter Leiner

Yanik E et al. Variation in cancer incidence among patients with ESRD during kidney function and nonfunction intervals. JASN. 2015; online 12. November. doi: 10.1681/ ASN.2015040373.

\title{
Steinentfernung bei rezidivierenden Harnwegsinfekten
}

\begin{abstract}
Jeder zweite Patient mit rezidivierenden Harnwegsinfekten und asymptomatischen Nierensteinen ist nach der Steinentfernung von erneuten Infektionen befreit. Doch wer genau profitiert von dem Eingriff?
\end{abstract}

U $\mathrm{m}$ die Frage zu beantworten, ob rezidivierende Harnwegsinfekte durch die Entfernung der Nierensteine eingedämmt werden können, werteten Mohamed Omar et al., Cleveland, USA, retrospektiv die Daten von 120 Patienten aus, die sich einer operativen Steinentfernung unterzogen hatten. $32 \%$ wurden mit Stoßwellenlithotripsie behandelt, $7 \%$ per Ureteroskopie und $61 \%$ per perkutaner Nephrolithotomie. Postoperativ waren alle Patienten steinfrei. Sie litten vor dem Eingriff unter rezidivierenden Harnwegsinfekten und nicht obstruktiven, asymptomatischen Nierensteinen. Vor der chirurgischen Intervention wurden alle antibiotisch behandelt. Bei $48 \%$ wa- ren bis zu einer mittleren Nachbeobachtungszeit von 14 Monaten nach der Steinentfernung keine Harnwegsinfektionen mehr aufgetreten. Bei $52 \%$ kehrten die Infektionen auch nach dem Eingriff zurück. Als signifikante Risikofaktoren hierfür zeigten sich eine bestehende Hochdruckerkrankung, ein Diabetes mellitus bei Männern sowie eine schwarze Ethnie. Außerdem waren Infektionen mit Enterokokken hartnäckiger als ausschließlich durch Escherichia coli ausgelöste. Die Art des Nierensteins und die Methode der Entfernung hatten keinen Einfluss. Als unabhängige Risikofaktoren für anhaltende Infektionen auch nach Steinentfernung ergaben sich Infek- tionen mit Escherichia coli, eine Hypertonie sowie eine schwarze Ethnie. Eine Chinolonresistenz lag präoperativ bei $\mathrm{Pa}$ tienten mit rezidivierenden Harnwegsinfekten häufiger vor (48 \% vs. $27 \%$ ) als bei denen, welche die Steinentfernung von ihren Harnwegsinfekten befreite. $82 \%$ der Patienten mit fortgesetzten Infektionen waren nach der Steinentfernung mit dem gleichen Keim infiziert wie zuvor.

Fazit: Da Escherichia coli im Vergleich zu anderen Bakterien am besten eradiziert werden konnte, empfehlen Omar et al. vor allem Patienten mit rezidivierenden Escherichia-coli-Infektionen einen operativen Eingriff zur Entfernung nachgewiesener Nierensteine anzubieten. Patienten, die entsprechende Risikofaktoren für rezidivierende Infektionen auch nach Steinentfernung haben, sollten auf dieses Problem hingewiesen werden.

Dr. Christine Starostzik

Omar $M$ et al. Does stone removal help patients with recurrent urinary tract infections? J Urol. 2015;194(4):997-1001. 